\title{
Multiple User Profile Merging (MUPE): Key Challenges for Environment Awareness
}

\author{
Ben Salem and Matthias Rauterberg \\ Department of Industrial Design, \\ Technische Universiteit Eindhoven, P.O. Box 513, 5600 MB Eindhoven, NL \\ \{b.i.salem, g.w.m.Rauterberg\} atue.nl
}

\begin{abstract}
In this paper we describe the issues that need to be addressed when setting up an aware environment occupied simultaneously by several users. Combining the delivery of services for various users simultaneously requires the setting up of users profiles as a record of their needs, requirements and desires. We are thus interested in a assessment of the requirements and specifications of user profiling. Furthermore there is also a need for the merger of multiple user profiles. As we are involved in the development of a smart family home and a responsive Exhibition booth, we will investigate user profiling and profile management within these two contexts. We finally discuss some issues that we consider detrimental to the success of aware environments.
\end{abstract}

\section{Introduction}

An aware and responsive environment is one that addresses user needs, requirements and desires (NRDs) by delivering an experience based on a dialogue and some understanding between the environment and the user(s). Such an environment is seen as engaging the user and reacting to his actions.

In the case of several users in the same environment, the environment could respond to each user individually or it could responds to users as a cohesive group. It is interesting to note the shift of paradigm. From one of the inhabitant or visitor of the environment, to one of the user of the environment. In the context of a smart home, not only will the user inhabit the house but they will interact with is as if it were a system running an application. In this instance the environment's application is no less than servicing the users and responding to their NRDs. This is indeed no small challenge.

\subsection{Applications}

We aim to develop an aware environment for two applications: one is an aware family home and the other is a smart exhibition space. In both case we are interested in a responsive environment that deliver an experience that can be positively qualified by the users. It is challenging to precisely describe what experience we would like to deliver, especially in the context of the smart home. For the aware exhibition space, it is much easier to do so, as we aim to develop an exhibition space that is attractive, 
entertaining, informative, responsive and agreeable to the users/visitors. For the sake of clarity we will illustrate this paper with examples from the aware family home.

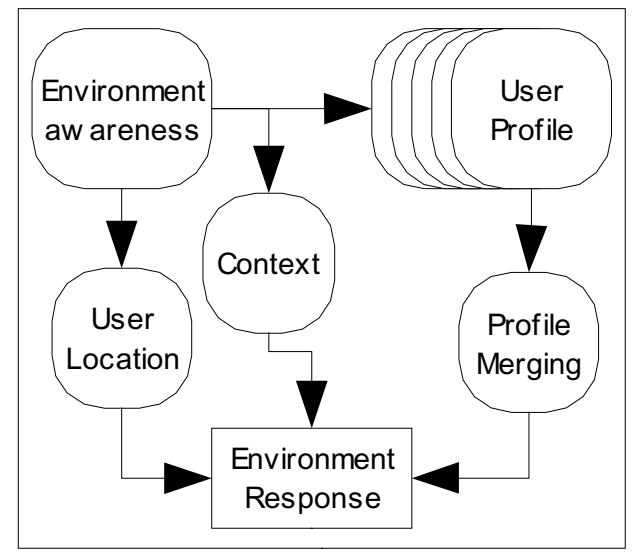

Fig. 1. From awareness to response

\subsection{Environment Awareness}

Environment awareness for multiple users is based on (1) the environment detection and observation of individual users, (2) the merging and combination of multiple user NRDs, (3) the resolution of any conflicts that might occur, shall they be of resources (e.g. one TV set for all the family) or of interest (father wants children to watch specific program) and, (4) the environment adaptation to the users as a cohesive group and as individuals.

\subsection{User Location}

Within the environment, the user location is essential for localised and personalised service delivery. Knowing where the user is, at a particular time, will ensure that the environment is not perceived as dumb (e.g. turning on all the lights in the house simply because someone opened the main door). The more personalised the service delivery is, for example the selection of TV channels, the more refined the user location detection needs to be.

\subsection{Context}

The service delivery context helps refine the perception by the user of an awareness of the environment. Time and concurrence of events help improve user experience (e.g. watching TV at night implies a lower sound volume) and improve user profiles (e.g. always listen to radio while drinking coffee). 


\section{Profiles}

In our opinion the environment awareness depends on the information the system has of the users. Such information is continuously collected and used to construct user profiles. Individual profiles are then refined and updated each time the users are within the environment or when further data is fed to the environment. This emphasis the importance of the environment being aware of its users. Indeed, in the context of profiling, the environment awareness is useful for two purposes. First to continuously update user profiling that is then fed to the environment responses to the users actions. Second, to localise users, and deliver a response that might be personalised, localised or specialised.

User profiles were suggested as an improvement for a variety of applications. From query enhancement (Korfhage, 1984) and digital libraries (Amato, 1999), to the personalisation of websites (Goel, 2002) and, enhanced interpersonal communication (Lukose, 2003). Current trends are for the integration of user profiling in the delivery of services for an aware environment such as Familly Interactive TV (Goren-Bar, 2003), or exhibitions (Kraemer, 2002).

We have run a survey of patents in the area of user profile and have compiled the following table.

Table 1. Review of patents

\begin{tabular}{ll}
\hline \multicolumn{1}{c}{ Patent \# } & \multicolumn{1}{c}{ Topic } \\
\hline US2004141003 & $\begin{array}{l}\text { Maintaining a user interest profile reflecting } \\
\text { changing interests of a customer } \\
\text { Comp2004128156 } \\
\text { Sources }\end{array}$ \\
WO2004055745 & $\begin{array}{l}\text { User Profile Portability } \\
\text { Predicting content choices by searching a profile } \\
\text { database }\end{array}$ \\
GB2396934 & $\begin{array}{l}\text { Personalised profile update } \\
\text { Method, system and program product for identify- } \\
\text { ing similar user profiles in a collection }\end{array}$ \\
TW566039 & $\begin{array}{l}\text { System for providing personalized services } \\
\text { Recommendation of video content based on the } \\
\text { user profile of users with similar viewing habits }\end{array}$ \\
\hline
\end{tabular}

\subsection{Profiles}

Profiles are a set of characteristics and properties that describe attributes, behaviour, and rules of engagement. A profile is defined as a formal summary or analysis of data, representing distinctive features or characteristics (ref. dictionary.com). A profile can be used to describe either single or group of elements. In our current context elements could be individuals, services, products, or systems. We are focusing on profiles for two homogeneous sets of elements, specifically: Services and Users. 


\subsection{Service Profiles}

A service profile includes the explicit attributes and meta-data of the service being delivered such as theme, name, brand, model, genre etc. The options, the operational requirements, and the availability of the services are also part of this type of profile.

\subsection{User Profiles}

A user profile is a combination of user identity, interests and NRDs. There is a decrease of importance in the NRDs from the needs to the requirements and then the desires. Needs are the most important user specifications as they are essential (e.g. sleeping). Requirements are necessary for a normal activity (e.g. Telephone). As for desires, they are the least important user specifications. The User profile contains user preferences regarding the service to be offered. This will reduce or eliminate all together the otherwise necessary dialogue between the environment and the user to specify some options and parameters. In the case of a simultaneous presence of several users in the environment, a merger algorithm must be implemented and be adaptive and reactive to users changing interests. To add some difficulties such changes in interest can be detected either from group or individual behaviours.

A user profile consists of a set of specifications, characteristics and parameters that describe the user and his NRDs. The profile contains as well information about the user habits, preferences and traits. Finally the profile includes the user role, privileges and status.

The creation and the management of a user profile is based on three parameters; (1) The user history and past NRDs as provided for example by a questionnaire when a new service will be offered, or when a service require specific information. (2) The general and implicit user preferences as generated from the observation of the user behaviour and finally. (3) The more explicit user feedback as provided by the user actions and responses on the service delivery.

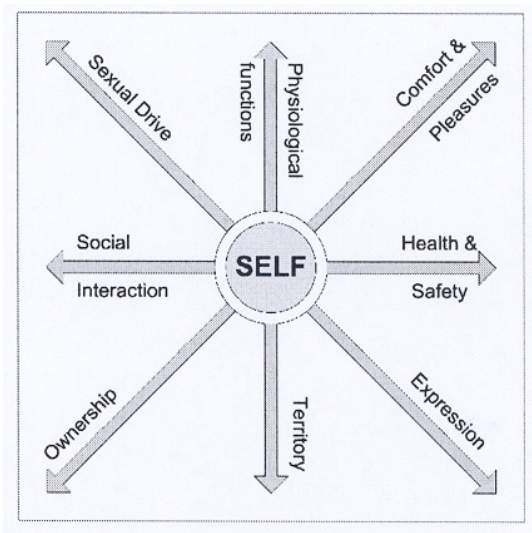

Fig. 2. Human needs 


\subsection{Mapping of Profiles}

There are three profile mapping scenarios. A one-to-one mapping (1:1), a one-tomany mapping (1:M), and a many-to-many mapping (M:N). In the case of (1:1) mapping there is no need for profile merging, as there is no conflict (either of resources or of interests). In all other cases merging is an essential and an un-resolved issue.

Table 2. Mapping of profiles and necessary mergers

\begin{tabular}{lll}
\hline & \multicolumn{1}{c}{$1: \mathrm{M}$} & \multicolumn{1}{c}{ M:N } \\
\hline Service to User & User Profiles Merger & Both Profiles Mergers \\
User to Service & Service Profiles Merger & Both Profiles Mergers \\
User to User & User Profiles Merger & User Profiles Mergers \\
\hline
\end{tabular}

\section{Merging Profiles}

Our aim is to develop a method that addresses the issue of merging multiple profiles and the resolution of resources and services conflicts within the context of an aware responsive and adaptive environment. We do not intend to develop a profile technique or an aware environment per se. Our objectives are complementary to such endeavours, but we are focusing on the issues related to the processing of profiles when multiple users are using/present in an aware environment.

We propose the merging the profiles of the multiple users of the environment because we want to relate each user to the environment. Such relation can be between one user or between several users and the environment. In the case of several users there could be a concurrent or a separate relation. The environment responsiveness depends on the processing of user profile and the delivery by the environment of services and supports in-accordance with some rule of engagement. In this context it would be useful to determine the common features and trends that characterise the environment users. This would ensure a more effective and probably efficient operation of the environment, and if all users share a same need then that need will be given high priority. We see a co-relation between the commonality of profile features and the importance and priority of environment response.

There a need to integrate the user profiles in the awareness of the environment, but there is also a need for a merging of the users' profiles to ensure a cohesive environment response.

\subsection{Why Merging?}

The user profile merger is used by the environment to either (1) modify and influence the environment response to the users, (2) or to concurrently respond to the users, (3) or finally to direct an environment request to the users.

The first case occurs when there is neither a conflict of resources or a conflict of interest. The second case happens when there is a conflict of resources and finally the third case is for situations when there is a conflict of interest. A conflict of resources is typically about sharing some facilities or services between users such as one TV in 
the home. A conflict of interest is when some user(s) want to have influence over other user(s).

We are concerned with profile merging and management to gain from the environment awareness what are the users trends, traits and habits. Such a merging would also help highlight and rank common and individual characteristics. It is all done with the aim of delivering an experience that is at least matching if not going further than the users expectations. Another issue is the timing of the user profiling. It could be Real-Time, delayed or off-line. There is no one fit all timing and depending on the circumstances either a real-time user profiling occurs especially when there is explicit user input while for other cases a delayed or even off-line profiling occurs. The later timing suitable for example in the initial setting of a service.

\subsection{Merging Techniques}

The merging techniques that we will use are novel and are based on the statistical analysis of vector distribution in the meta-data space. Currently there are three merging techniques that could be used: Boolean logic, Vector space model and probabilistic model (Chen, 2000) and we wish to further improve as well as combine them. Collaborative filtering systems have also been used (Kohrs, 2000), (Ko, 2003).

Boolean logic is based on the merging of the profile by similarity reinforcement of the profile parameters and at the same time the mutual exclusion of conflicting parameters. The Boolean logic has it limitations, as the weighting of the parameters is difficult to include. The vector space model has more potential for weighting. Essentially each parameter of the user profile is associated with a dimension in a vector space. The weighting is translated into a coordinate along each dimension. Limitations of this technique lie in the lack of scope for predictability and merging. Finally the probabilistic model, which relates to the assessment of the frequency of occurrence of a parameter, has limitation in that there is not always a correlation between the frequency and the importance of a parameter (e.g. One of the user has diabetes).

We propose to investigate a vector space model combined with a feedback mechanism based on the comparison of predicted and actual users behaviour and environment response to correct the vector describing the merged profiles $\left(V_{m p}\right)$. In other words, if the actual vector $V_{m p}$ value is different from the predicted $V_{m p}^{\prime}$ then a correction occurs and is fed back to the environment awareness.

The value of $V_{m p}^{\prime}$ is predicted from the likelihood of occurrence of events, responses and behaviours. This likelihood is evaluated by comparing in the time domain discrete series of $V_{m p}$ values. As a result one is expecting a learning curve in the environment's awareness and the need for frequent corrections at the initial stages of the system.

\subsection{Selection of Metrics}

There are many metrics we could use for the calculation of disparity between predicted $V_{m p}^{\prime}$ and actual $V_{m p}$. We will focus on three, namely Euclidean, Mahalanobis and Battacharyah. The choice of metrics is made according to the level of co-relation and co-variance, if any, there might be between the different dimensions of the $V_{m p}$. One of the most important issue to address is the dimension reduction, in particular the elimination of redundant or irrelevant dimensions. Reducing the dimensions of the vectors will ensure the faster processing of the data. 


\section{Environment Response: Services and Experience}

The User profile must include information pertinent to several domains related to the quality of experience (QoE) and the Quality of Services (QoS) each user will be expecting from the aware environment. he profiling of users we are relying on is based on the continuous monitoring of user position, actions, and behaviour. The QoE depends on the variety and comprehensiveness of the options available as well as the granularity of the profiles. The QoE also depends on the solving of conflict of interests between users as well between different requirements within the same user profile. As for the QoS, it depends on the services supported by the environment and the refresh rate of the actualisation of the environment awareness. It is also linked to the richness of the user profiles and the effectiveness and efficiency of the profile merger.

There could be conflict between the QoE and the QoS. As the environment services become more comprehensive and efficient there is a risk that the environment becomes far too adaptive and responsive to the user. What we mean is that the environment could behave like the user's genie fulfilling all his wishes and desires. There is a need to define a domain and a protocol of responsiveness of the environment. Otherwise we could deliver an environment that is so finely tuned to the user that all the random experiences, part of the events of a normal life, are removed. As if the user was living in a sensory deprivation chamber.

Similarly the QoE needs to be sufficiently "entertaining" and "interesting" to deliver an experience of the environment that avoids the "living in a box" syndrome. We define entertaining as being hospitable and care taking. As for interesting we understand it as being stimulating and intellectually involving. There is a challenging balance to strike, as too much involvement from the user would defeat the purpose of an aware environment.

The responsiveness of the environment relies on the right selection of behaviour cues and user instructions. Ultimately there is a design decision about who would be in charge of the Services and the experience. Is it the user of the environment, the designer of the system or the environment itself? Furthermore, our opinion is that the environment should play different roles from a reserved housemaid localised and at attention to a Gaia (goddess of earth), as in a holistic service embedded in the fabric of the environment. Intermediate roles would be a more intruding butler and an interactive agent.

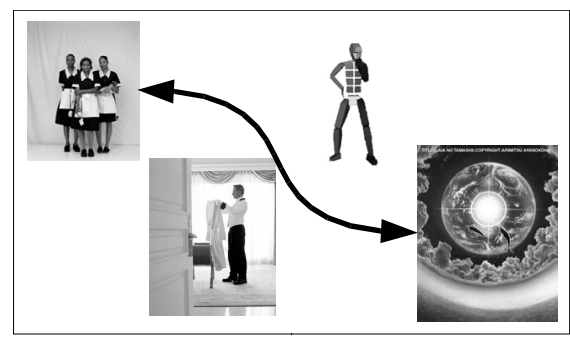

Fig. 3. From Housemaid to Gaia (pictures from internet) 
We must also keep this in mind as the ultimate aware environment description borrows much from the realms of Science Fiction.

\section{Open Issues}

As part of this project we are seeking the optimum rate and domain of adaptation of the aware environment. This would ensure we process the right information from the user profiles and merge them to obtain a cohesive and useful set of NRDs applicable to the environment. We also would like to define the level bandwidth and domain of control the users have over the environment. Is the user's experience similar to a dialogue, a menu navigation, or a more demanding and intense interaction. How direct is the interaction between the user and his environment is also relevant. The directness of the interface has detrimental effects on the QoE, it could be based on switches, remote control, token, interface, speech. The duration of the interaction tasks (continuous, discrete, contextual, triggered) will also influence the users experience.

\subsection{Conflicts}

Our understanding of conflicts is that of occurrences when there is either a limited set of resources available, there is a contradictory set of NRDs, or there is an overlapping set of NRDs.

In the first instance the resources limitation can be addressed. The environment can be enhanced with more high-in-demand resources. Some of the conflicts can be easily resolved when they involve desires or to a certain extent requirements. The environment can either make a decision based on the ranking of the desires and requirements as available in the user profiles or could assess the comparative ranking of the users (e.g. The father desires come before the children's). However difficulties arise when desires or requirements are contradictory or overlapping. In the first instance there is a conflict between the users NRDs and it is the environment function to avoid this turning into a conflict between users. Two metaphors could be used the intrusive butler or the reserved housemaid. With the intrusive butler the system will assess the history of the NRDs in conflict and if for example there is a track record of regular occurrence and fulfilment of one of the NRDs then it would take precedence. In the case of a reserved housemaid configuration the environment will suggest the fulfilment of the various NRDs using extra resources such as a different room or in the case of the TV programme to record on VCR one of the alternative to be shown later. As a last resort suggesting a relocation for the users could avoid them entering into conflict.

\subsection{Semantic Aspect}

Another issue related to the profile merging is the weighting of the importance and significance of each user's profiles and parameters. 


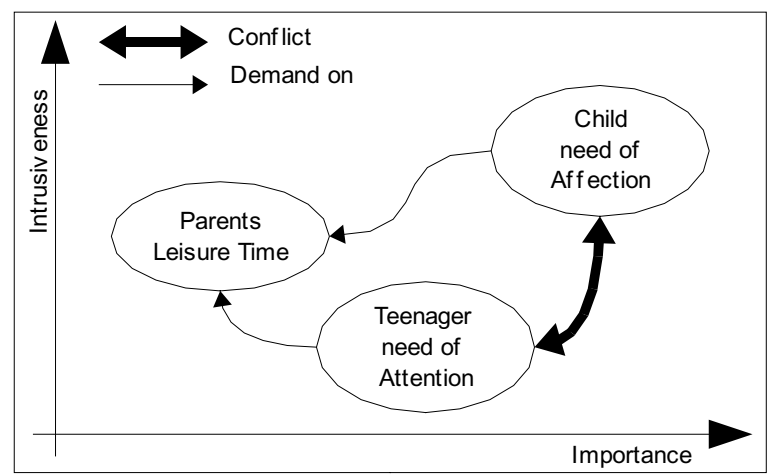

Fig. 4. Tensions between profiles

Within the same domain how is the weighting and classification achieved? As an example, is the father choice of TV programme overwhelmingly more important than the children's one? When the domains are different is the classification and weighting achieved using the ranking of users. For example is the parents need for intimacy higher than the children need for bedtime stories?

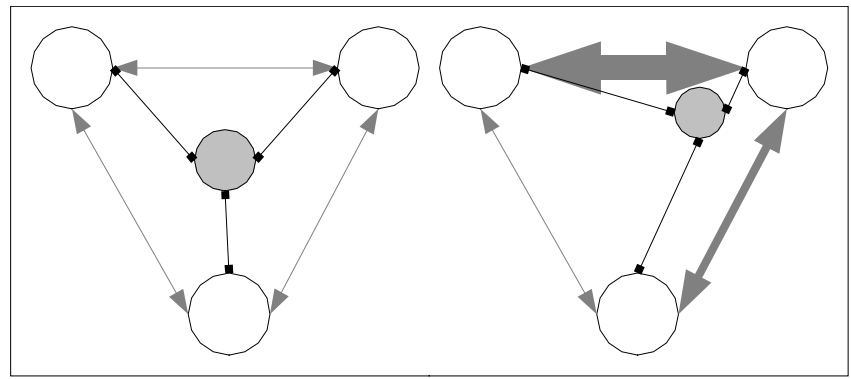

Fig. 5. Tension Grading

We call these weightings and classifications tension grades and there are dependant on the user identity and role in the environment, the domain of the user parameter and the user own importance given to that parameter. As illustrated in figure 5 the tension grading modifies the outcome of the profile merger. Without weighting the merged Vector is a geometric average of the combined profiles. This is not the case with the weighting even in a weighting as simple as ranking the different profile parameters in order of importance.

\subsection{User Push or Environment Pull}

Deciding who is in charge of the user profiling and the profile merger is a fundamental question for this project. In other words is the paradigm applied one of user's push or one of environment's pull. This apply also to the adaptation rate of the environment as well as the service delivery. We could also argue that the inter-actions between user(s) and environment are also subject to this paradigm choice. 


\subsection{Resonance}

The proper adaptation behaviour of an adaptive system depends on the individual user profile in a particular context of use with a particular application. If two adaptive systems (in our case the human being and the adaptive home appliance) are coupled with each other, the following aspects have to be taken into account: (1) the adaptation rate ' $A$ ' of the environment as a system ' $\mathrm{s}$ ' to the user's behaviour $\left(\mathrm{R}_{\mathrm{sA}}\right)$, and (2) the two different kinds of human ' $h$ ' influences on the system: (a) the explicit control rate ' $C$ ' based on direct user input to the system $\left(R_{h C}\right)$ and (b) the implicit adaptation rate ' $A$ ' by the user to the system's output $\left(\mathrm{R}_{\mathrm{hA}}\right)$. The main challenge of designing such a coupling is to avoid an unintended acceleration and/or mismatch between both subsystems based on a closed loop coupling. How should be the optimal relation $\left[\mathrm{R}_{\mathrm{hC}}\right.$ $\left.+\mathrm{R}_{\mathrm{hA}}\right] \leftrightarrow\left[\mathrm{R}_{\mathrm{sA}}\right]$ established? What is the proper balance between $\left[\mathrm{R}_{\mathrm{hC}}\right] \leftrightarrow\left[\mathrm{R}_{\mathrm{hA}}\right]$ ? Even more challenging, in the context of a multi-user environment the adaptive system has to resolve the possible trade-off between all present user profiles.

\section{Research Strategy}

Single user profiling technology is already available; the main interest of this project is to extend these approaches to multiple-user profile merging.

We have divided our project into the following phases:

- Investigation of various frameworks and prototypes for user-profiling architectures and software.

- Definition of an architecture for multi-user-profiles merging.

- Installation of different MUPEs for two different applications in a home environment and in an exhibition space, integration and test.

- Data collection with several groups of test subjects for validation.

- Data analysis and extraction of significant and relevant parameters, which could be used for assessing the quality of the implemented MUPEs.

- Improving and optimising one MUPE as final delivery.

\subsection{Project Outcome}

We will deliver expertise in the area of designing a MUPE within the context of an aware home and an exhibition booth. Prototype installations for two different MUPEs will be available during the project; at the end an optimised version will be operational. Data gathering and analysis software will be developed to provide semantically relevant input to adaptive appliances and services. Deliverables include different working systems, which could be used for other projects, composed of what would have been determined as efficient and effective combination and integration of single user profiling techniques. This project would be relevant transition and evolution from the expertise built-up by us in the area of our research in the area of automatic mental model evaluation (AMME) which focuses on the analysis of behavioural data describing the interaction between users and systems in learning situations (Rauterberg, 1993). 


\subsection{Industrial Relevance}

Interests on this project have been expressed to enhance the adaptivity for specific appliances in a smart home and a responsive environment. We have two major companies interested in this project, and we are cooperating with them to further define and specify the aim, scope and content of the MUPE project.

\section{Conclusion}

We would like to establish expertise in the selection of relevant information about users. One should know what to collect and how. Are the user desires, needs, requirements to be considered, and what is the importance of the users' behaviour. The Multiple User mErger (MUPE) system is a combination, an overlapping and a classification of different user profile parameters. We propose to develop a methodology and the technologies necessary for the completion of a MUPE system. Finally how reliable is the profiling and the management and merging proposed is an issue we would like to address.

We hope to have clearly stated what are the challenges and issues related to profile merging within the context of an aware environment.

\section{References}

1. Amato, G, Straccia, U, User Profile Modelling and Applications to Digital Libraries, In ECDL '99, LNCS 1696, Abiteboul,S, Vercoustre, A-M,(Eds.), 184-197.

2. Chen, P-M, Kuo, F-C, An Information retrieval system based on a user profile, Journal of Systems and Software, 54(2000)3-8.

3. Goel, M, Sarkar, S, Web Site Personalization Using User Profile Information, In AH 2002, LNCS 2347, De Bra, P, Brusilovsky, P, Conejo, R, (Eds.), 510-513.

4. Goren-Bar, D, Glinansky, O, FIT-recommend ing TV programs to family members, Computer \& Graphics, 28, 2004, 149-156.

5. Ko, S.-J., Group Typical Preference Extraction Using Collaborative Filtering Profile, In EC-Web 2003, LNCS 2738, Bauknecht, K., Min Tjoa, A., Quirchmayr, G., (Eds.), pp. 414-423, 2003.

6. Kohrs, A., Merialdo, B., Using category-based collaborative liftering in the active webmuseum, In Proceedings of the IEEE Intl. Conf. on Multimedia - Vol. 1, 2000.

7. Korfhage, Query Enhancement by User Profiles, In Proc. of the joint BCS \& ACM Symposium on Research \& Development in Information Retrieval, 1984, 111-121.

8. Kraemer, R, Schwander, P, Bluetooth based wireless Internet applications for indoor hot spots: experience of a successful experiment during CeBIT 2001, Computer Networks, 41, 2003, 303-312.

9. Lukose, RJ, Adar, E, Tyler, JR, Sengupta, C, SHOCK: Communicating with Computational messages and Automatic Private Profiles, In Proc. WWW 2003, May 20-24, 2003, Budapest, 291-300.

10. Rauterberg, M., AMME: an Automatic Mental Model Evaluation to analyze user behaviour traced in a finite, discrete state space, Ergonomics, vol. 36(11), 1993, pp. 1369-1380. 